\title{
Análise fitossociológica de um fragmento de Floresta Estacional Decidual: Parque Estadual do Turvo, RS
}

\author{
Rafaelo Balbinot ${ }^{1}$, Fernanda Raquel Lambrecht ${ }^{1 *}$, Fabio Marcelo Breunig ${ }^{1}$, Jonathan William Trautenmüller ${ }^{2}$, Lênio Soares Galvão ${ }^{3}$, \\ Luciano Denardi ${ }^{1}$, Rafael Vendruscolo ${ }^{1}$
}

\begin{abstract}
${ }^{1}$ Universidade Federal de Santa Maria, Linha 7 de Setembro, s/n, BR 386 Km 40, CEP 98400-000, Frederico Westphalen, RS, Brasil ${ }^{2}$ Universidade Federal do Paraná, Av. Prefeito Lothário Meissner, 3400, CEP 80210-170, Curitiba, PR, Brasil

${ }^{3}$ Instituto Nacional de Pesquisas Espaciais, Av. dos Astronautas, 1758, CEP 12245970, São José dos Campos, SP, Brasil
\end{abstract}

"Autor correspondente:

fernanda.lambrecht@hotmail.com

Termos para indexação:

Inventário florestal

Diversidade florística

Yucumã

Index terms:

Forest inventory

Floristic diversity

Yucumã

\begin{abstract}
Resumo - O ecossistema formado pela Floresta Estacional Decidual (FED) predomina na região do Alto Uruguai no Estado do Rio Grande do Sul, Brasil. O presente trabalho teve como objetivo analisar de forma descritiva a composição florística e fitossociológica dos componentes arbóreos de um fragmento de FED presente no Parque Estadual do Turvo (PET), localizado no Município de Derrubadas, RS (floresta do Yucumã). Utilizou-se o método de área fixa, sendo instaladas 18 unidades amostrais de $1.000 \mathrm{~m}^{2}$ de forma aleatória, orientadas de acordo com a estrada principal no parque. Todos os indivíduos arbóreos com diâmetro a 1,30 m do solo (DAP) superior a $10 \mathrm{~cm}$ foram medidos e identificados. Verificou-se a presença de 842 indivíduos, distribuídos em 32 famílias botânicas, 67 gêneros e 83 espécies (12\% não foram identificados). As famílias que apresentaram maior riqueza de espécies foram Fabaceae, Euphorbiaceae, Meliaceae, Myrtaceae e Sapindaceae. O Índice de Shannon estimado foi de 3,72.
\end{abstract}

\section{Phytosociology analysis of a fragment of Seasonal Deciduous Forest: Parque Estadual do Turvo, RS, Brazil}

Historico do artigo:

Aprovado em 04/04/2016

Publicado em 30/06/2016

doi: $10.4336 / 2016 . p f b .36 .86 .837$

\begin{abstract}
The ecosystem formed by the Seasonal Deciduous Forest (SDF) predominates in the region of Alto Uruguay in Rio Grande do Sul State, Brazil. This study aimed to analyze descriptively the floristic composition and the phytosociology of trees from a fragment of SDF present in Parque Estadual do Turvo (PET) located in the Municipality of Derrubadas, Rio Grande do Sul State (Yucumã forest). We used the method of fixed area, based on 18 sample units with $1,000 \mathrm{~m}^{2}$ installed randomly oriented by the main road of the park. All wood species with diameter at $1.3 \mathrm{~m}$ above ground level $(\mathrm{DBH})$ greater than $10 \mathrm{~cm}$ were measured and identified. It was observed the presence of 842 individuals belonging to 32 families, 67 genera and 83 species (12\% were not identified). The families with the highest number of species were Fabaceae, Euphorbiaceae, Meliaceae, Myrtaceae and Sapindaceae. Shannon index estimated was 3.72 .
\end{abstract}

\section{Introdução}

As florestas estacionais são as mais representativas da cobertura florestal do Rio Grande do Sul, predominando na região do Alto Uruguai, ao longo das encostas-sul da formação Serra Geral e Leste do Planalto Sul-RioGrandense ou Serra do Sudeste (Scipioni et al., 2011).

No domínio do Bioma Mata Atlântica, as Florestas
Estacionais distribuem-se principalmente nas regiões interiores, distantes da margem oceânica dominada pela Floresta Ombrófila Densa. A distribuição da Floresta Estacional Decidual (FED), aparentemente, segue padrões dependentes da precipitação e da temperatura (Oliveira-Filho \& Fontes, 2000) e, em segundo plano, de fatores como geologia e geomorfologia, que influenciam a distribuição dos canais de drenagem, e também das 
características pedológicas (Oliveira-Filho \& Ratter, 2002; Giehl \& Jarenkow, 2008).

A FED é caracterizada por atributos estruturais relacionados à perda parcial das folhas (caducifólia), condicionada à sazonalidade climática. A deciduidade das folhas é resultado de fatores climáticos restritivos, como o frio e o menor fotoperíodo durante o inverno, ocorrendo especialmente nas plantas do dossel superior e emergentes, podendo atingir mais de $50 \%$ das espécies (IBGE, 2012).

É possível identificar quatro formações distintas da FED (Kray \& Jarenkow, 2003): Aluvial, Terras Baixas, Submontana e Montana. As florestas aluviais ocorrem quase exclusivamente nas bacias dos rios do RS, constituídas, em sua maioria, por espécies higrófitas deciduais, como, por exemplo, o açoita-cavalo (Luehea divaricata). As formações de terras baixas ocorrem em latitudes entre 24 e $32^{\circ} \mathrm{Sul}$, na faixa altimétrica de 5 a 30 m. Em sua maior formação está a submontana, em que aparecem principalmente os gêneros Parapiptadenia, Apuleia e Peltophorum. A formação montana situa-se entre 400 e $1.000 \mathrm{~m}$ de altitude, entre as latitudes $24 \mathrm{e}$ $32^{\circ} \mathrm{Sul}$ (IBGE, 2012).

Conforme o Inventário Florestal Contínuo do Rio Grande do Sul (Rio Grande do Sul, 2002), a área coberta por florestas naturais no estado coresponde a $17,53 \%$, sendo que a FED abrange uma área de 1.176.245 ha, o que corresponde a $4,16 \%$ da cobertura florestal do estado. A área preservada no Parque Estadual do Turvo (PET) é um dos poucos remanescentes desta vegetação original (Wallauer \& Albuquerque, 1986). A floresta do PET se estende para a província de Missiones, com mais de 2 milhões de ha (Bulfe, 2008). O PET é refúgio também para muitas espécies da fauna, especialmente para grandes mamíferos, como onças-pintadas, porcosdo-mato, antas, dentre outros (Bertoletti et al., 1989; Baptista \& Leal-Zanchet, 2010).

Embora seja reconhecida a importância da área preservada no PET, até o momento são encontrados poucos estudos relacionados à vegetação (Ruschel et al., 2005, 2006). Os trabalhos publicados concentraram-se em levantamentos da composição florística, com uma pequena quantidade de parcelas (menos de 15). Portanto, é importante a realização de novas pesquisas, para atualizar e incrementar as informações relacionadas à vegetação deste remanescente.

Desse modo, o objetivo do presente trabalho é analisar de forma descritiva a composição florística e fitossociológica do componente arbóreo de um fragmento de FED presente no PET, em Derrubadas, RS (floresta do Yucumã).

\section{Material e métodos}

\section{Área de estudo}

O presente trabalho foi realizado em um fragmento de FED no PET (Figura 1), com cerca de 17.500 ha. O Parque situa-se no noroeste do estado, entre as coordenadas $27^{\circ} 07^{\prime}$ a $27^{\circ} 16^{\prime}$ Sul e $53^{\circ} 48^{\prime}$ a $54^{\circ} 04^{\prime}$ Oeste, no município de Derrubadas, RS, junto ao rio Uruguai, fazendo divisa com o estado de Santa Catarina e a província Argentina de Missiones (Rio Grande do Sul, 2005).

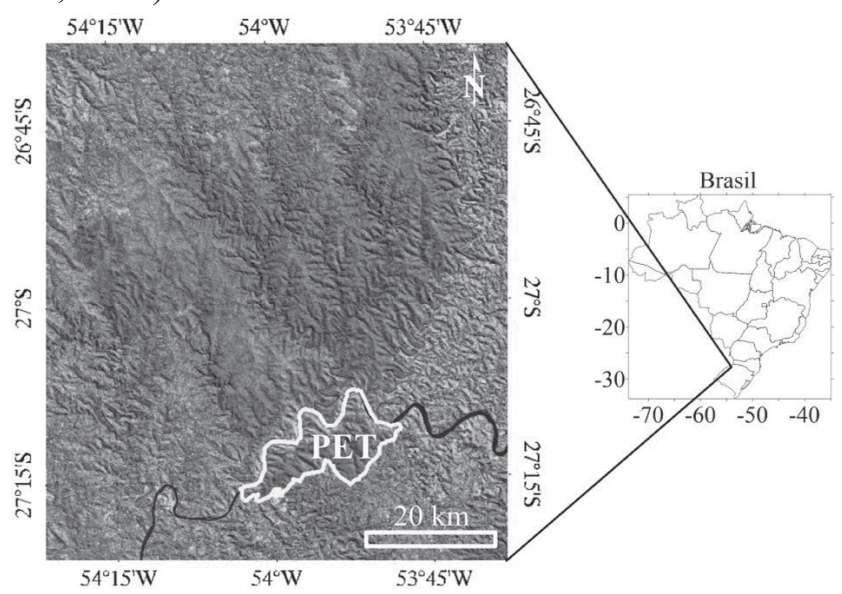

Figura 1. Localização geográfica do Parque Estadual do Turvo (PET) no noroeste do Rio Grande do Sul. Imagem OLI/Landsat -8 .

O clima da região é Subtropical muito úmido, com regime de chuvas equilibrado, com média anual entre $1.700 \mathrm{~mm}$ e $1.900 \mathrm{~mm}$ e temperatura média entre $20{ }^{\circ} \mathrm{C}$ e $23{ }^{\circ} \mathrm{C}$, com redução da precipitação no inverno (Rossato, 2014).

\section{Levantamento de campo}

Para o estudo da vegetação, utilizou-se o método de área fixa, sendo instaladas 18 unidades amostrais (UA) de $1.000 \mathrm{~m}^{2}(20 \mathrm{~m} \times 50 \mathrm{~m})$ de forma aleatória, orientadas pelo trajeto da estrada principal que corta o parque. As parcelas foram implantadas a partir da abertura de um eixo central e orientação por meio de cruzeta e nível. Todos os indivíduos arbóreos com diâmetro a $1,30 \mathrm{~m}$ do solo (DAP) maior que $10 \mathrm{~cm}$ foram medidos e identificados. 


\section{Análise dos dados}

$\mathrm{Na}$ análise dos dados utilizou-se planilha eletrônica. A análise florística (gênero, espécie e família) e a determinação dos parâmetros fitossociológicos para a estrutura horizontal (densidade, dominância, frequência e índice de valor de importância) foram determinados de acordo com Lamprecht (1990). O índice de valor de cobertura foi calculado conforme Foerster (1973).

O sistema de classificação botânica adotado para angiospermas foi o proposto por Angiosperm Phylogeny Group (Angiosperm Phylogeny Group, 2009). Para as gimnospermas utilizou-se a classificação proposta por Christenhusz et al. (2011). Os nomes científicos das espécies foram verificados na Lista de Espécies da Flora do Brasil 2014 (Reflora, 2015) e Missouri Botanical Garden (Tropicos, 2015).

\section{Resultados e discussão}

A suficiência amostral do fragmento inventariado foi testada pela curva espécie-área (ou curva de acumulação de espécies). Observou-se grande número de novas espécies nas parcelas iniciais, reduzindo gradativamente. Foi observada tendência à estabilidade da curva a partir de $16.000 \mathrm{~m}^{2}$ amostrados. De acordo com Longhi et al. (1999), a tendência à estabilização é suficiente para representar a vegetação em florestas naturais. Hüller et al. (2011) encontraram tendência à estabilização da curva espécie-área em $2.400 \mathrm{~m}^{2}$, em estudo realizado no Parque natural municipal de Santo Ângelo, RS. No entanto, a estabilização pode ocorrer apenas após levantamento em área maior, como demonstrado por Silva \& Scariot (2004), que observaram tendência à estabilização quando atingiram $6.000 \mathrm{~m}^{2}$ de unidades amostrais quantificadas, havendo poucas espécies novas inclusas com o aumento da área amostral, em trabalho realizado em áreas de afloramentos de calcário na região da Bacia do Rio Paraná no Município de São Domingos, Goiás.

No fragmento estudado, foram amostrados 842 indivíduos com DAP superior a $10 \mathrm{~cm}$, dos quais 9 estavam mortos. Os indivíduos foram distribuídos em 32 famílias botânicas, 67 gêneros e 83 espécies, sendo que nove espécies não foram identificadas. Esses resultados estão de acordo com os valores reportados por Ruschel et al. $(2005,2006)$ que realizaram estudos com indivíduos com DAP $\geq 5 \mathrm{~cm}$, trabalho realizado no PET, usando o método de ponto de quadrante central. Esses autores observaram 37 famílias e 78 espécies.
$\mathrm{Na}$ Tabela 1, estão apresentados os resultados de estudos fitossociológicos realizados em outros fragmentos no estado, demonstrando uma grande variedade de espécies. Os trabalhos em Frederico Westphalen (Scipioni et al., 2011) e Tenente Portela (Dias et al., 1992) apresentaram resultados semelhantes ao deste trabalho. No entanto, em Santa Tereza (Vaccaro et al., 1999) e Jaguari (Hack et al., 2005) os autores obtiveram resultados inferiores em termos de diversidade arbórea. Porém, com este aspecto comparativo, fica evidente a necessidade de mais pesquisas sobre a florística e fitossociologia em outras regiões do estado com ocorrência da FED. Na Tabela 2 pode-se verificar a relação das espécies, ordenadas por famílias, com os respectivos nomes científicos e nomes vulgares (ou comuns).

Segundo Magurran (1989), as medidas de diversidade têm sido frequentemente utilizadas como indicadores do bom funcionamento dos ecossistemas. O conceito de diversidade relaciona a riqueza de espécies, isto é, o número de espécies de uma comunidade, e a uniformidade (abundância), que representa a distribuição do número de indivíduos por espécie. Isto pode ser observado através do Índice de Shannon. Verificou-se no fragmento alta diversidade, onde o Índice de Shannon estimado foi de 3,72, concordando com o valor de 3,73 reportado por Ruschel et al. (2006) para a mesma área de estudo. Em estudos com fragmentos florestais próximos do PET, Scipioni et al. (2011) verificaram valor de 3,49. Longhi et al. (1999) obtiveram índice de 3,12. Estes valores foram superiores aos encontrados por Vaccaro (1997) para o estágio de sucessão secundária da Floresta Estacional Decidual $\left(H^{\prime}=2,72\right)$ e por Nascimento et al. (2000) para fragmento florestal na região Central do Estado $\left(H^{\prime}=2,90\right)$. Desse modo, podemos observar que a vegetação apresentou uma distribuição mais uniforme do número de indivíduos em relação ao número de espécies e consequentemente, alta diversidade de espécies para a área de estudo. Tendo em vista que geralmente os valores encontrados variam de 1,5 a 3,5 e raramente são acima de 5. A alta diversidade encontrada no presente estudo pode estar relacionada à conservação que vem sendo mantida no Parque.

As famílias que apresentaram maior número de indivíduos foram Arecaceae (72,2 indivíduos ha-1), Euphorbiaceae $\left(29,4\right.$ indivíduos ha $\left.{ }^{-1}\right)$, Lauraceae $\left(27,2\right.$ indivíduos ha-1 $^{-1}$ e Fabaceae (24,4 indivíduos ha-1). 
Tabela 1. Resultados de estudos fitossociológicos em Floresta Estacional Decidual no RS.

\begin{tabular}{|c|c|c|c|}
\hline Autor & Método & $\begin{array}{l}\text { Dados referente ao } \\
\text { levantamento }\end{array}$ & Local \\
\hline Hack et al. (2005) & $\begin{array}{l}14 \text { unidades amostrais de } 10 \mathrm{~m} \text { x } 100 \mathrm{~m} \\
\left(1.000 \mathrm{~m}^{2}\right) \text {, demarcadas de maneira sistemática, } \\
\text { com total de } 1,4 \text { ha de área de estudo }\end{array}$ & $\begin{array}{l}592 \text { indivíduos no fragmento } \\
\text { amostrado, } 28 \text { famílias } \\
\text { botânicas, } 45 \text { gêneros, } 54 \\
\text { espécies arbustivo-arbóreas }\end{array}$ & Jaguari, RS \\
\hline Vaccaro et al. (1999) & $\begin{array}{l}\text { área fixa e processo de amostragem estratificada, } \\
\text { com distribuição sistemática das unidades } \\
\text { amostrais, em } 10 \text { parcelas, totalizando } 2.000 \mathrm{~m}^{2}\end{array}$ & $\begin{array}{l}786 \text { árvores (floresta } \\
\text { secundária), } 23 \text { famílias, } 39 \\
\text { gêneros, } 48 \text { espécies }\end{array}$ & Santa Tereza, RS. \\
\hline Dias et al. (1992) & $\begin{array}{c}\text { quadrantes, } 107 \text { pontos em } 6 \text { linhas paralelas em, } \\
\text { aproximadamente, } 2,6 \text { ha }\end{array}$ & $\begin{array}{l}546,59 \text { indivíduos por ha, } \\
37 \text { famílias, } 69 \text { gêneros, } 88 \\
\text { espécies }\end{array}$ & $\begin{array}{l}\text { Tenente Portela, RS } \\
\text { (PET) }\end{array}$ \\
\hline Scipioni et al. (2011) & $\begin{array}{l}10 \text { parcelas permanentes de } 20 \mathrm{~m} \text { x } 50 \mathrm{~m} \text {, } \\
\text { distribuídas de forma aleatória, constituída por } \\
10 \text { subparcelas quadradas de } 100 \mathrm{~m} 2 \text {, totalizando } \\
1 \text { ha }\end{array}$ & $\begin{array}{l}660 \text { indivíduos por ha, } 31 \\
\text { famílias, } 69 \text { gêneros, } 79 \\
\text { espécies }\end{array}$ & $\begin{array}{l}\text { Frederico Westphalen, } \\
\text { RS (Submontana) }\end{array}$ \\
\hline Presente estudo & $\begin{array}{l}\text { área fixa, } 18 \text { unidades amostrais de } \\
20 \mathrm{~m} \text { x } 50 \mathrm{~m}\left(1.000 \mathrm{~m}^{2}\right) \text { de forma aleatória, } \\
\text { orientadas pelo trajeto da estrada, total de } 1,8 \text { ha }\end{array}$ & $\begin{array}{l}\text { 467,78 indivíduos por ha, } 32 \\
\text { famílias botânicas, } 67 \text { gêneros, } \\
83 \text { espécies }\end{array}$ & Derrubadas, RS (PET) \\
\hline
\end{tabular}

As famílias que se destacaram em riqueza de espécies foram Fabaceae, com quatorze espécies, seguida de Euphorbiaceae, Meliaceae, Myrtaceae, e Sapindaceae, com quatro espécies cada. As famílias Boraginaceae, Lauraceae, Rutaceae e Salicaceae tiveram três espécies cada.

Das espécies identificadas, nenhuma delas esteve presente em todas as unidades amostrais, sendo as de maiores frequências: Syagrus romanzoffiana (17), Nectandra megapotamica (12), Chrysophyllum gonocarpum (11), Balfourodendron riedelianum (10), Chrysophyllum marginatum (9), Cedrella fissilis (9) e Maclura tinctoria (9). Tais espécies contribuíram com $23,20 \%$ da frequência relativa total. Os resultados dos parâmetros fitossociológicos podem ser observados na Tabela 3, ordenados pelo valor de importância (IVI) das espécies. Ruschel et al. (2005) observaram, dentre as mais frequentes em todas as florestas remanescentes, em um estudo que incluía parte do PET, $N$. megapotamica, C. gonocarpum, C. marginatum e $B$. riedelianum, semelhante a este trabalho, além das espécies Ocotea diosyirifolia e Cabralea canjerana.

Em relação à densidade absoluta total de 467,78 indivíduos ha ${ }^{-1}$ estimada para a comunidade arbórea, cabe ressaltar que este é um valor considerado como mediano. Hack et al. (2005) observaram resultado semelhante $(422,8$ indivíduos ha-1), em estudo realizado em FED no município de Jaguari, RS, em que demarcaram, de maneira sistemática, 14 unidades amostrais de $10 \mathrm{~m}$ x $100 \mathrm{~m}$, sendo incluídos os indivíduos com circunferência à altura do peito $(\mathrm{CAP})=30$. Por outro lado, Longhi et al. (2000) encontraram um valor bem superior $\left(853,4\right.$ indivíduos $\left.\mathrm{ha}^{-1}\right)$ em estudo em FED no município de Santa Maria, onde utilizaram 16 unidades amostrais de $10 \mathrm{~m} \times 20 \mathrm{~m}$, distribuídas de forma sistemática, seguindo a mesma orientação da declividade, com o mesmo critério da inclusão $(\mathrm{CAP} \geq 30 \mathrm{~cm})$.

As espécies que apresentaram maior densidade no fragmento foram S.romanzoffiana (72,2 indivíduos $\left.\mathrm{ha}^{-1}\right)$, Sebastiania commersoniana (29,4 indivíduos ha-1), N. megapotamica (27,2 indivíduos ha $\left.^{-1}\right)$, L. muehlbergianus (24,4 indivíduos ha-1 ${ }^{-1}$ e Eugenia subterminalis $\left(16,6\right.$ indivíduos ha $\left.{ }^{-1}\right)$. Estas espécies contribuíram com $36,3 \%$ do número total de árvores mensuradas, sendo que $S$. romanzoffiana contribuiu com $15,4 \%$. 
Tabela 2. Relação das espécies amostradas em fragmento de Floresta Estacional Decidual no Parque Estadual do Turvo, RS.

\begin{tabular}{|c|c|c|}
\hline Família & Nome Científico & Nome Comum \\
\hline Achatocarpaceae & Achatocarpus praecox Griseb. & Quebra-machado \\
\hline Anonnaceae & Annona sp. & Ariticum \\
\hline \multirow{2}{*}{ Apocynaceae } & Tabernaemontana catharinensis DC. & Jasmim-catavento \\
\hline & Aspidosperma australe Müll. Arg. & Quebracho \\
\hline \multirow[t]{2}{*}{ Araliaceae } & Schefflera morototoni (Aubl.) Maguire, Steyerm. \& Frodin & Caixeta \\
\hline & Aralia warmingiana (Marchal) J. Wen & Cinamomo-do-mato \\
\hline Arecaceae & Syagrus romanzoffiana (Cham.) Glassman & Coqueiro \\
\hline Asteraceae & Gochnatia polymorpha (Less.) Cabrera & Cambará \\
\hline Bignoniaceae & Jacaranda micrantha Cham. & Carobão \\
\hline \multirow{3}{*}{ Boraginaceae } & Cordia americana (L.) Gottshling \& J. E. Mill. & Guajuvira \\
\hline & Cordia trichotoma (Vell.) Arrab. ex Steud. & Louro \\
\hline & Cordia ecalyculata Vell. & Louro-mole \\
\hline \multirow[t]{2}{*}{ Cannabaceae } & Celtis sp. & Celtis \\
\hline & Trema micrantha $(\mathrm{L}$.$) Blume$ & Trema \\
\hline Cardiopteridaceae & Citronella paniculata (Mart.) Howard & Citronela \\
\hline Caricaceae & Jacaratia spinosa (Aubl.) DC. & Paineira \\
\hline \multirow{4}{*}{ Euphorbiaceae } & Sebastiania commersoniana (Baill.) L. B. Sm. \& Downs & Branquilho \\
\hline & Tetrorchidium rubrivenium Poepp. \& Endl. & Embirão \\
\hline & Gymnanthes concolor Spreng. & Laranjeira-do-mato \\
\hline & Alchornea triplinervia (Spreng.) M. Arg. & Tanheiro \\
\hline \multirow{14}{*}{ Fabaceae } & Holocalyx balansae Micheli & Alecrim \\
\hline & Albizia edwallii (Hoehne) Barneby \& J. Grimes & Angico-branco \\
\hline & Parapiptadenia rigida (Benth.) Brenan & Angico-vermelho \\
\hline & Myrocarpus frondosus Allemão & Cabreúva \\
\hline & Peltophorum dubium (Spreng.) Taub. & Canafístula \\
\hline & Gleditsia amorphoides (Griseb.) Taub. & Coronilha \\
\hline & Erythrina falcata Benth. & Corticeira-da-serra \\
\hline & Lonchocarpus muehlbergianus Hassl. & Farinha-seca \\
\hline & Apuleia leiocarpa (Vogel) J. F. Macbr. & Grápia \\
\hline & Inga marginata Willd. & Ingá \\
\hline & Bauhinia forficata Link & Pata-de-vaca \\
\hline & Lonchocarpus campestris Mart. ex Benth. & Rabo-de-bugio \\
\hline & Machaerium stipitatum (DC.) Vogel & Sapuva \\
\hline & Enterolobium contortisiliquum (Vell.) Morong & Timbaúva \\
\hline \multirow[t]{2}{*}{ Lamiaceae } & Vitex megapotamica (Spreng.) Moldenke & Tarumã \\
\hline & Nectandra lanceolata Nees & Canela-amarela \\
\hline \multirow[t]{2}{*}{ Lauraceae } & Ocotea puberula $($ Rich.) Nees & Canela-guaicá \\
\hline & Nectandra megapotamica (Spreng.) Mez & Canela-preta \\
\hline Malvaceae & Luehea divaricata Mart. \& Zucc. & Açoita-cavalo \\
\hline
\end{tabular}


Tabela 2. continuação.

\begin{tabular}{|c|c|c|}
\hline Família & Nome Científico & Nome Comum \\
\hline \multirow{4}{*}{ Meliaceae } & Cabralea canjerana (Vell.) Mart. & Canjerana \\
\hline & Guarea macrophylla Vahl & Catiguá-morcego \\
\hline & Trichilia clausseni D. DC. & Catinguá \\
\hline & Cedrella fissilis Vellozo & Cedro \\
\hline Monimiaceae & Hennecartia omphalandra J. Poiss. & Cega-olho \\
\hline \multirow{3}{*}{ Moraceae } & Sorocea bonplandii (Baill) W. C. Burger & Cincho \\
\hline & Ficus sp. & Ficus \\
\hline & Maclura tinctoria (L.) Don ex Steud. & Tajuva \\
\hline \multirow{4}{*}{ Myrtaceae } & Eugenia subterminalis DC. & Cambuí-pitanga \\
\hline & Campomanesia xanthocarpa $\mathrm{O}$. Berg & Guabiroba \\
\hline & Calyptranthes tricona D. Legrand & Guamirim \\
\hline & Plinia trunciflora (O. Berg) Kausel & Jabuticaba \\
\hline Nyctaginaceae & Pisonia zapallo Griseb. & Anzol-de-lontra \\
\hline Phytolacaceae & Phytolacca dioica $\mathrm{L}$. & Umbú \\
\hline Primulaceae & Myrsine umbellata Mart. & Capororoca \\
\hline Rosaceae & Prunus myrtifolia (L.) Urb. & Pesegueiro-do-mato \\
\hline Rubiaceae & Randia ferox (Cham. \& Schltdl.) DC. & Limoeiro-do-mato \\
\hline \multirow{4}{*}{ Rutaceae } & Helietta apiculata Benth. & Canela-de-veado \\
\hline & Pilocarpus pennatifolius Lem. & Cutia \\
\hline & Balfourodendron riedelianum Engler & Guatambú \\
\hline & Zanthoxylum rhoifolium Lam. & Mamica-de-cadela \\
\hline \multirow{3}{*}{ Salicaceae } & Banara tomentosa $\mathrm{Clos}$ & Banara \\
\hline & Casearia sylvestris $\mathrm{Sw}$. & Carvalhinho \\
\hline & Xylosma pseudosalzmannii Sleumer & Sucará \\
\hline \multirow{4}{*}{ Sapindaceae } & Matayba elaeagnoides Radlk. & Camboatá-branco \\
\hline & Cupania vernalis Cambess. & Camboatá-vermelho \\
\hline & Allophylus edulis (St. Hil.) Radlk. & Chal-chal \\
\hline & Diatenopteryx sorbifolia Radlkofer & Maria-preta \\
\hline \multirow{2}{*}{ Sapotaceae } & Chrysophyllum gonocarpum (Mart. \& Eichler ex Miq.) Engl. & Aguaí-da-serra \\
\hline & Chrysophyllum marginatum (Hook. \& Arn.) Radlk. & Aguaí-leitero \\
\hline \multirow{2}{*}{ Solanaceae } & Solanum mauritianum Scop. & Fumeiro-bravo \\
\hline & Solanum sanctaecatharinae Dunal & Joa-manso \\
\hline Styracaceae & Styrax leprosus Hook. \& Arn. & Carne-de-vaca \\
\hline Urticaceae & Urera baccifera (L.) Gaudich. ex Wedd. & Urtigão \\
\hline
\end{tabular}


Tabela 3. Parâmetros fitossociológicos das espécies arbóreas encontradas em um fragmento de Floresta Estacional Decidual no Parque Estadual do Turvo, Derrubadas, RS.

\begin{tabular}{|c|c|c|c|c|c|c|c|c|c|}
\hline Nome científico & $\mathbf{N}$ & DA & DR & DoA & DoR & FA & FR & IVI & IVC \\
\hline Syagrus romanzoffiana & 130 & 72,22 & 15,44 & 1,66 & 8,12 & 0,52 & 5,12 & 28,68 & 23,56 \\
\hline Nectandra megapotamica & 49 & 27,22 & 5,82 & 1,75 & 8,54 & 0,37 & 3,61 & 17,97 & 14,36 \\
\hline Lonchocarpus muehlbergianus & 44 & 24,44 & 5,23 & 1,24 & 6,07 & 0,25 & 2,41 & 13,70 & 11,29 \\
\hline Sebastiania commersoniana & 53 & 29,44 & 6,29 & 0,45 & 2,20 & 0,15 & 1,51 & 10,00 & 8,49 \\
\hline Peltophorum dubium & 5 & 2,78 & 0,59 & 1,24 & 6,06 & 0,15 & 1,51 & 8,16 & 6,65 \\
\hline Diatenopteryx sorbifolia & 15 & 8,33 & 1,78 & 0,79 & 3,88 & 0,09 & 0,90 & 6,57 & 5,66 \\
\hline Cabralea canjerana & 17 & 9,44 & 2,02 & 0,73 & 3,59 & 0,25 & 2,41 & 8,02 & 5,61 \\
\hline Maclura tinctoria & 21 & 11,67 & 2,49 & 0,60 & 2,94 & 0,28 & 2,71 & 8,14 & 5,43 \\
\hline Eugenia subterminalis & 30 & 16,67 & 3,56 & 0,23 & 1,14 & 0,06 & 0,60 & 5,30 & 4,70 \\
\hline Chrysophyllum gonocarpum & 22 & 12,22 & 2,61 & 0,39 & 1,91 & 0,34 & 3,31 & 7,84 & 4,53 \\
\hline Inga marginata & 27 & 15,00 & 3,21 & 0,24 & 1,18 & 0,25 & 2,41 & 6,80 & 4,39 \\
\hline Cordia trichotoma & 12 & 6,67 & 1,43 & 0,58 & 2,86 & 0,22 & 2,11 & 6,39 & 4,28 \\
\hline Ficus sp. & 9 & 5,00 & 1,07 & 0,58 & 2,82 & 0,22 & 2,11 & 5,99 & 3,88 \\
\hline Chrysophyllum marginatum & 17 & 9,44 & 2,02 & 0,32 & 1,56 & 0,28 & 2,71 & 6,29 & 3,58 \\
\hline Alchornea triplinervia & 10 & 5,56 & 1,19 & 0,49 & 2,39 & 0,12 & 1,20 & 4,78 & 3,57 \\
\hline Holocalyx balansae & 7 & 3,89 & 0,83 & 0,56 & 2,74 & 0,22 & 2,11 & 5,68 & 3,57 \\
\hline Cedrella fissilis & 10 & 5,56 & 1,19 & 0,42 & 2,06 & 0,28 & 2,71 & 5,95 & 3,24 \\
\hline Parapiptadenia rigida & 5 & 2,78 & 0,59 & 0,52 & 2,54 & 0,15 & 1,51 & 4,64 & 3,14 \\
\hline Trichilia clausseni & 17 & 9,44 & 2,02 & 0,19 & 0,95 & 0,22 & 2,11 & 5,07 & 2,97 \\
\hline Tetrorchidium rubrivenium & 11 & 6,11 & 1,31 & 0,32 & 1,54 & 0,22 & 2,11 & 4,96 & 2,85 \\
\hline Balfourodendron riedelianum & 13 & 7,22 & 1,54 & 0,25 & 1,21 & 0,31 & 3,01 & 5,76 & 2,75 \\
\hline Vitex megapotamica & 2 & 1,11 & 0,24 & 0,51 & 2,51 & 0,06 & 0,60 & 3,35 & 2,75 \\
\hline Pisonia zapallo & 14 & 7,78 & 1,66 & 0,22 & 1,07 & 0,19 & 1,81 & 4,54 & 2,73 \\
\hline Allophylus edulis & 15 & 8,33 & 1,78 & 0,18 & 0,90 & 0,22 & 2,11 & 4,79 & 2,68 \\
\hline Jacaranda micrantha & 8 & 4,44 & 0,95 & 0,35 & 1,73 & 0,22 & 2,11 & 4,79 & 2,68 \\
\hline Sorocea bonplandii & 18 & 10,00 & 2,14 & 0,10 & 0,50 & 0,19 & 1,81 & 4,45 & 2,64 \\
\hline Luehea divaricata & 11 & 6,11 & 1,31 & 0,23 & 1,14 & 0,19 & 1,81 & 4,25 & 2,45 \\
\hline Helietta apiculata & 7 & 3,89 & 0,83 & 0,28 & 1,35 & 0,09 & 0,90 & 3,09 & 2,18 \\
\hline Cordia americana & 6 & 3,33 & 0,71 & 0,27 & 1,31 & 0,09 & 0,90 & 2,93 & 2,02 \\
\hline Calyptranthes tricona & 11 & 6,11 & 1,31 & 0,13 & 0,63 & 0,12 & 1,20 & 3,14 & 1,93 \\
\hline Annona sp. & 8 & 4,44 & 0,95 & 0,19 & 0,91 & 0,09 & 0,90 & 2,77 & 1,86 \\
\hline Jacaratia spinosa & 5 & 2,78 & 0,59 & 0,24 & 1,17 & 0,12 & 1,20 & 2,97 & 1,77 \\
\hline Erythrina falcata & 3 & 1,67 & 0,36 & 0,27 & 1,31 & 0,09 & 0,90 & 2,57 & 1,66 \\
\hline Ocotea puberula & 8 & 4,44 & 0,95 & 0,13 & 0,62 & 0,15 & 1,51 & 3,08 & 1,57 \\
\hline Cordia ecalyculata & 8 & 4,44 & 0,95 & 0,12 & 0,58 & 0,15 & 1,51 & 3,03 & 1,53 \\
\hline Albizia edwallii & 4 & 2,22 & 0,48 & 0,21 & 1,01 & 0,12 & 1,20 & 2,69 & 1,48 \\
\hline
\end{tabular}


Tabela 3. Continuação.

\begin{tabular}{|c|c|c|c|c|c|c|c|c|c|}
\hline Nome científico & $\mathbf{N}$ & DA & DR & DoA & DoR & FA & FR & IVI & IVC \\
\hline Casearia sylvestris & 8 & 4,44 & 0,95 & 0,09 & 0,42 & 0,15 & 1,51 & 2,87 & 1,37 \\
\hline Apuleia leiocarpa & 5 & 2,78 & 0,59 & 0,15 & 0,72 & 0,06 & 0,60 & 1,91 & 1,31 \\
\hline Machaerium stipitatum & 8 & 4,44 & 0,95 & 0,07 & 0,33 & 0,09 & 0,90 & 2,19 & 1,28 \\
\hline Phytolacca dioica & 4 & 2,22 & 0,48 & 0,14 & 0,70 & 0,06 & 0,60 & 1,78 & 1,18 \\
\hline Gochnatia polymorpha & 5 & 2,78 & 0,59 & 0,09 & 0,42 & 0,09 & 0,90 & 1,92 & 1,01 \\
\hline Campomanesia xanthocarpa & 6 & 3,33 & 0,71 & 0,05 & 0,23 & 0,12 & 1,20 & 2,15 & 0,94 \\
\hline Prunus myrtifolia & 2 & 1,11 & 0,24 & 0,14 & 0,70 & 0,06 & 0,60 & 1,54 & 0,94 \\
\hline Banara tomentosa & 5 & 2,78 & 0,59 & 0,07 & 0,34 & 0,09 & 0,90 & 1,84 & 0,94 \\
\hline Styrax leprosus & 3 & 1,67 & 0,36 & 0,12 & 0,58 & 0,06 & 0,60 & 1,53 & 0,93 \\
\hline Plinia trunciflora & 3 & 1,67 & 0,36 & 0,12 & 0,57 & 0,03 & 0,30 & 1,23 & 0,93 \\
\hline Cupania vernalis & 4 & 2,22 & 0,48 & 0,08 & 0,38 & 0,06 & 0,60 & 1,46 & 0,86 \\
\hline Schefflera morototoni & 3 & 1,67 & 0,36 & 0,08 & 0,39 & 0,09 & 0,90 & 1,65 & 0,75 \\
\hline Tabernaemontana catharinensis & 4 & 2,22 & 0,48 & 0,05 & 0,25 & 0,06 & 0,60 & 1,33 & 0,73 \\
\hline Achatocarpus praecox & 3 & 1,67 & 0,36 & 0,07 & 0,32 & 0,03 & 0,30 & 0,98 & 0,68 \\
\hline Pilocarpus pennatifolius & 4 & 2,22 & 0,48 & 0,03 & 0,12 & 0,09 & 0,90 & 1,50 & 0,60 \\
\hline Zanthoxylum rhoifolium & 2 & 1,11 & 0,24 & 0,06 & 0,28 & 0,06 & 0,60 & 1,12 & 0,51 \\
\hline Bauhinia forficata & 3 & 1,67 & 0,36 & 0,03 & 0,15 & 0,09 & 0,90 & 1,40 & 0,50 \\
\hline Matayba elaeagnoides & 3 & 1,67 & 0,36 & 0,03 & 0,14 & 0,09 & 0,90 & 1,40 & 0,49 \\
\hline Enterolobium contortisiliquum & 1 & 0,56 & 0,12 & 0,07 & 0,34 & 0,03 & 0,30 & 0,76 & 0,46 \\
\hline Urera baccifera & 2 & 1,11 & 0,24 & 0,05 & 0,22 & 0,06 & 0,60 & 1,06 & 0,46 \\
\hline Celtis sp. & 3 & 1,67 & 0,36 & 0,02 & 0,08 & 0,09 & 0,90 & 1,34 & 0,44 \\
\hline Gymnanthes concolor & 3 & 1,67 & 0,36 & 0,02 & 0,08 & 0,09 & 0,90 & 1,34 & 0,43 \\
\hline Aspidosperma australe & 2 & 1,11 & 0,24 & 0,03 & 0,16 & 0,06 & 0,60 & 1,00 & 0,40 \\
\hline Myrocarpus frondosus & 1 & 0,56 & 0,12 & 0,04 & 0,19 & 0,03 & 0,30 & 0,61 & 0,31 \\
\hline Guarea macrophylla & 2 & 1,11 & 0,24 & 0,01 & 0,07 & 0,06 & 0,60 & 0,91 & 0,31 \\
\hline Trema micrantha & 2 & 1,11 & 0,24 & 0,01 & 0,07 & 0,06 & 0,60 & 0,91 & 0,31 \\
\hline Lonchocarpus campestris & 1 & 0,56 & 0,12 & 0,04 & 0,19 & 0,03 & 0,30 & 0,61 & 0,31 \\
\hline Gleditsia amorphoides & 1 & 0,56 & 0,12 & 0,04 & 0,17 & 0,03 & 0,30 & 0,59 & 0,29 \\
\hline Aralia warmingiana & 1 & 0,56 & 0,12 & 0,02 & 0,09 & 0,03 & 0,30 & 0,51 & 0,21 \\
\hline Hennecartia omphalandra & 1 & 0,56 & 0,12 & 0,01 & 0,06 & 0,03 & 0,30 & 0,48 & 0,18 \\
\hline Nectandra lanceolata & 1 & 0,56 & 0,12 & 0,01 & 0,06 & 0,03 & 0,30 & 0,48 & 0,18 \\
\hline Solanum mauritianum & 1 & 0,56 & 0,12 & 0,01 & 0,05 & 0,03 & 0,30 & 0,47 & 0,17 \\
\hline Randia ferox & 1 & 0,56 & 0,12 & 0,01 & 0,04 & 0,03 & 0,30 & 0,46 & 0,16 \\
\hline Myrsine umbellata & 1 & 0,56 & 0,12 & 0,01 & 0,04 & 0,03 & 0,30 & 0,46 & 0,16 \\
\hline Citronella paniculata & 1 & 0,56 & 0,12 & 0,01 & 0,04 & 0,03 & 0,30 & 0,46 & 0,16 \\
\hline Solanum sanctaecatharinae & 1 & 0,56 & 0,12 & 0,01 & 0,03 & 0,03 & 0,30 & 0,45 & 0,15 \\
\hline Xylosma pseudosalzmannii & 1 & 0,56 & 0,12 & 0,001 & 0,02 & 0,03 & 0,30 & 0,44 & 0,14 \\
\hline
\end{tabular}

$\mathrm{DA}=$ densidade absoluta (indivíduos ha $\left.\mathrm{a}^{-1}\right) ; \mathrm{DR}=$ densidade relativa (\%); DoA $=$ dominância absoluta $\left(\mathrm{m}^{2} \mathrm{ha}^{-1}\right)$; DoR $=$ dominância relativa $(\%)$; $\mathrm{FA}=$ frequência absoluta $(\%) ; \mathrm{FR}=$ frequência relativa $(\%) ; \mathrm{IVI}=$ índice de valor de importância (\%); IVC = índice de valor de cobertura $(\%)$. 
As espécies que apresentaram maior frequência relativa no fragmento (Tabela 3), foram $S$. romanzoffiana $(5,1 \%), N$. megapotamica $(3,6 \%)$, C. gonocarpum $(3,3 \%)$ e B. riedelianum (3,0\%). Farias et al. (1994) verificaram que $N$. megapotamica $(5,2 \%)$, Cupania vernalis $(5,18 \%)$ e Banara parviflora $(5,2 \%)$ foram as mais frequentes. De acordo com Lamprecht (1990), a frequência expressa a uniformidade de distribuição horizontal de cada espécie no terreno, caracterizando a sua ocorrência dentro das parcelas. Em relação à dominância, as espécies que se destacaram foram: N. megapotamica, S. romanzoffiana, L. muehlbergianus e Peltophorum dubium.

As dez espécies que apresentaram os maiores índices de valor de importância (IVI), contribuíram com $115,8 \%$ do IVI total, podendo ser observadas na Tabela 3. A densidade e a dominância foram os parâmetros que contribuíram para que $S$. romanzoffiana e $N$. megapotamica fossem as espécies de maior valor de importância e representassem juntas $46,7 \%$ do IVI total.

A distribuição por classe de diâmetro dos indivíduos arbóreos pode ser observada na Figura 2, onde $87,2 \%$ dos indivíduos estão presentes nas duas primeiras classes. A densidade populacional, bem como o número de indivíduos e sua distribuição nas classes de diâmetro, é um indicativo em relação à permanência da espécie na estrutura da floresta. Longhi et al. (1999), realizando estudo semelhante em um fragmento de FED em Santa Maria, RS, com 18 parcelas de $10 \mathrm{~m}$ x $20 \mathrm{~m}$, com limite de inclusão de árvores com CAP $>15 \mathrm{~cm}$, reportaram cerca de $42,2 \%$ dos indivíduos na classe com centro em $\mathrm{CAP}=20 \mathrm{~cm}$.

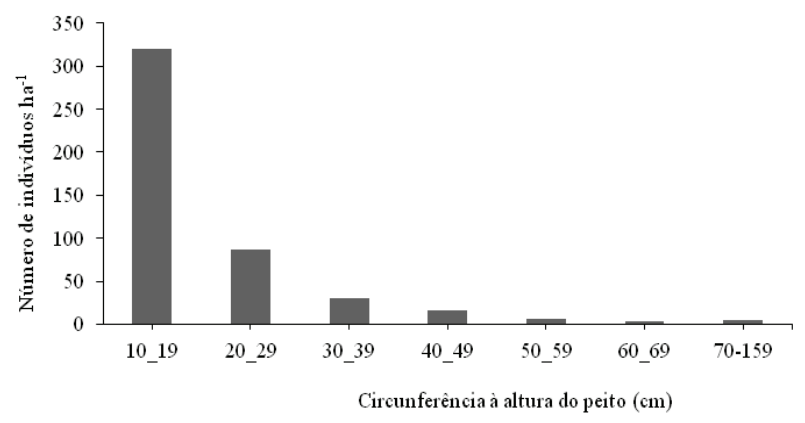

Figura 2. Distribuição por classe de diâmetro dos indivíduos arbóreos, amostrados em um fragmento de Floresta Estacional Decidual, Derrubadas, RS.

De acordo com Longhi et al. (1999), uma espécie que apresenta baixa densidade populacional tem maior possibilidade de ser substituída por outra no desenvolvimento da floresta, por razões naturais, ou em função das perturbações ocorridas na área. Observa- se que a maior frequência de indivíduos nas classes de menores diâmetros é característica de florestas inequiâneas, sendo a regeneração responsável por elevado número de indivíduos de pequeno porte e com pouca contribuição para a área basal total (Bianchin \& Bellé, 2013).

Considerando o índice de diversidade obtido $(3,72)$ e as características estruturais apresentadas pela floresta em estudo, é possível afirmar que o Parque Estadual do Turvo desempenha satisfatoriamente sua função de preservar o ecossistema da floresta decidual. Porém, notou-se um número reduzido de indivíduos de algumas espécies desta tipologia florestal, tais como Cedrela fissilis e Apuleia leiocarpa. Estas espécies apresentam baixo IVI, ocupando a $17^{\mathrm{a}}$ e a $38^{\mathrm{a}}$ posição na listagem, respectivamente, além de baixos valores de dominância (Tabela 3). A explicação para isto vem do fato que, apesar da manutenção da cobertura florestal da área do parque, houve exploração madeireira. Esta exploração está registrada no Plano de Manejo do PET (Rio Grande do Sul, 2005) e em depoimentos de antigos guardas do parque.

De acordo com a SEMA (Rio Grande do Sul, 2005) em toda a região havia retirada de madeira, inclusive do Parque, onde foram cortadas árvores de grande porte de forma clandestina. O sistema de corte de madeira utilizado naquela ocasião era chamado «ponta de lápis», no qual cortavam uma das extremidades da tora tal qual a ponta de um lápis e, em dias de chuva, nas regiões de encosta quando o solo ficava encharcado, a tora era deslizada até o rio Uruguai. A preferência na época era pela madeira de cedro, motivo pelo qual a espécie desapareceu da costa do Uruguai. Foi, então, substituída pela grápia, que passou a ser muito procurada, apesar do seu transporte pelo rio ser problemático, em função da densidade e do peso das toras.

Os resultados indicam que a estrutura fitossociológica do PET não corresponde à original e que ainda está se recuperando das intervenções realizadas no passado, possivelmente em consequência da exploração, que ocorreu principalmente em áreas próximas das estradas, onde foram alocadas as unidades amostrais deste estudo.

\section{Conclusões}

Apesar do reduzido número de parcelas utilizadas, foi possível verificar um grande número de espécies no fragmento, em relação a área total do Parque Estadual do Turvo. Recomenda-se que sejam realizados mais estudos 
no parque, com maior abrangência, por se tratar da maior área conservada do ecossistema da Floresta Estacional Decidual no estado do Rio Grande do Sul.

As espécies que mais se descaram foram Syagrus romanzoffiana, Nectandra megapotamica, Lonchocarpus muehlbergianus, Sebastiania commersoniana e Peltophorum dubium, representando 78,5\% do índice de valor de importância.

A pequena participação no IVI de espécies características da Floresta Estacional Decidual, como Cedrella fissilis e Apuleia leiocarpa, pode advir da exploração no passado, principalmente às margens das estradas, consequência que ainda se faz perceptível na fitossociologia do Parque Estadual do Turvo.

\section{Agradecimentos}

À Fundação de Amparo à Pesquisa do Estado de São Paulo (FAPESP) (Processo $n^{\circ}$ 2013/03908-3), ao Conselho Nacional de Desenvolvimento Científico e Tecnológico (CNPq) (Processo n ${ }^{\circ}$ 478085/2013-3) e à Fundação de Amparo à Pesquisa do Estado do Rio Grande do Sul (FAPERGS) (ARD - processo $n^{\circ}$ 12/0383-5 de 2012) pelo suporte financeiro para a execução do projeto.

\section{Referências}

The Angiosperm Phylogeny Group. An update of the Angiosperm Phylogeny Group classification for the orders and families of flowering plants: APG III. Botanical Journal of the Linnean Society, v. 161, p. 105-121, 2009. DOI: 10.1111/j.1095-8339.2009.00996.x.

Baptista, V. A. \& Leal-Zanchet, A. M. Land flatworm community structure in a Subtropical Deciduous Forest in Southern Brazil. Belgian Journal of Zoology, v. 140, p. 83-90, 2010.

Bianchin, J. E. \& Bellé, P. A. Fitossociologia e estrutura de um fragmento de Floresta Estacional Decidual Aluvial em Santa Maria - RS. Revista Agro@mbiente On-line, v. 7, n. 3, p. 322-330, 2013. DOI: 10.18227/1982-8470ragro.v7i3.1307.

Bertoletti, J. J. et al. Ictiofauna do Rio Uruguai superior entre os municípios de Aratiba e Esmeralda, Rio Grande do Sul, Brasil. Comunicações do Museu de Ciências e Tecnologia: Série Zoologia, v. 32, n. 48, p. 3-42, 1989.

Bulfe, N. M. L. Dinâmica de clareiras originadas de exploração seletiva deuma floresta estacional semidecidual na Provincia de Misiones, nordesteda Argentina. 2008. 73 f. Dissertação (Mestre em Ciências Florestais) - Universidade Federal do Paraná, Curitiba.

Christenhusz, M. J. M. et al. New Classification and linear sequence of extant gymnosperms. Phytotaxa, v. 19, n. 1, p. 19-55-70, 2011. DOI: 10.11646/phytotaxa.19.1.3.
Dias, L. L. et al. Levantamento florístico de uma área de mata subtropical no Parque Estadual do Turvo, Tenente Portela, RS. In: CONGRESSO NACIONAL SOBRE ESSÊNCIAS NATIVAS, 2., 1992, São Paulo. Anais. São Paulo: Instituto Florestal, 1992. p. 339-346.

Farias, J. A. C. et al. Estrutura fitossociológica de uma Floresta Estacional Decidual na região de Santa Maria, RS. Ciência Florestal, v. 4, n. 1, p. 109-128, 1994.

Foerster, M. Strukturanalysis eines tropischen regenwaldes in Kolumbien. Allgemeine Forest und Jagdzeitung, v. 144, p. 1-8, 1973.

Giehl, E. L. H. \& Jarenkow, J. A. Gradiente estrutural no componente arbóreo e relação com inundações em uma floresta ribeirinha, rio Uruguai, sul do Brasil. Acta Botânica Brasílica, v. 22, n. 3, p. 741753, 2008. DOI: 10.1590/S0102-33062008000300012.

Hack, C. et al. Análise fitossociológica de um fragmento de Floresta Estacional Decidual no município de Jaguari, RS. Ciência Rural, v. 35, n 5, p. 1083-1091, 2005. DOI: 10.1590/S010384782005000500015 .

Hüller, A. et al. Estrutura fitossociológica da vegetação arbórea do Parque Natural municipal de Santo Ângelo, Santo Ângelo, RS. Ciência Florestal, v. 21, n. 4, p. 629-639, 2011. DOI: 10.5902/198050984508.

IBGE. Manual técnico da vegetação brasileira. 2. ed. Rio de Janeiro, 2012. 271 p.

Kray, J. G. \& Jarenkow, J. A. Estrutura do componente arbóreo de mata estacional de encosta do Parque de Itapuã, Viamão, RS, 2003. In: CONGRESSO DE ECOLOGIA DO BRASIL, 6., 2003, Fortaleza. Anais... Fortaleza: Sociedade de Ecologia do Brasil, 2003. p. 452-453.

Lamprecht, H. Silvicultura nos trópicos. Hamburg: GTZ, 1990. $343 \mathrm{p}$.

Longhi, S. J. et al. Composição florística e estrutura da comunidade arbórea de um fragmento florestal no município de Santa MariaBrasil. Ciência Florestal, v. 9, n. 1, p. 115-133, 1999.

Longhi, S. J. et al. Aspectos fitossociológicos de fragmento de Floresta Estacional Decidual, Santa Maria, RS. Ciência Florestal, v. 10, n. 2, p. 59-74, 2000.

Magurran, A. E. Diversidad ecológica y su medición. Barcelona: Vedrà, 1989. $200 \mathrm{p}$.

Nascimento, A. R. T. et al. Análise da diversidade florística e dos sistemas de dispersão de sementes em um fragmento florestal na região central do Rio Grande do Sul, Brasil. Napaea, n. 12, p. 49$67,2000$.

Oliveira-Filho, A. T. \& Fontes, M. A. L. Patterns of floristic differentiation among Atlantic forests in southeastern Brazil and the influence of climate. Biotropica, v. 32, n. 4B, p. 793-810, 2000. DOI: 10.1111/j.1744-7429.2000.tb00619.x.

Oliveira-Filho, A. T. \& Ratter J. A. Vegetation physiognomies and woody flora of the cerrado biome. In: Oliveira, P. S.; Marquis, R. J. (Ed.). The Cerrado of Brazil: ecology and natural history of a neotropical savanna. New York: Columbia University Press, 2002. p. 91-120. 
Reflora: Lista de Espécies da Flora do Brasil. Rio de Janeiro: Jardim Botânico do Rio de Janeiro, [2015]. Disponível em: <http:// floradobrasil.jbrj.gov.br/>. Acesso em: 21 maio 2015.

Rio Grande do Sul. Secretaria Estadual do Meio Ambiente. Inventário florestal contínuo do Rio Grande do Sul. Porto Alegre: FATEC/SEMA, 2002. Disponível em: <www.ufsm.br/ifcrs > Acesso em: 18 fev. 2014.

Rio Grande do Sul. Secretaria Estadual do Meio Ambiente. Plano de Manejo do Parque Estadual do Turvo-RS. Porto Alegre. 2005. Disponível em: $<$ http://www.sema.rs.gov.br/upload/Plano_manejo_ PETurvo.pdf $>$. Acesso em: 17 jan. 2014.

Rossato, M. S. Os climas do Rio Grande do Sul: tendências e tipologias. In: Mendonça, F. (Org.). Os climas do Sul: em tempos de mudanças climáticas globais. Jundiaí: Paco, 2014. p. 217-271.

Ruschel, A. R. et al. Valuation and characterization of the timber species in remnants of the Alto Uruguay River ecosystem, southern Brazil. Forest Ecology and Management, v. 217, p. 103-116, 2005.
Ruschel, A. R. et al. Woody plant species richnessin the Turvo State Park, a large remnant of deciduous Atlantic Forest, Brazil. Biodiversity Conservation, v. 16, p. 1699-1714, 2006. DOI: 10.1007/s10531-006-9044-7.

Scipioni, M. C. et al. A fitossociologia em fragmento florestal no noroeste do estado do Rio Grande do Sul. Ciência Florestal, v. 21, n. 3, p. 409-419, 2011.

Silva, L. A. da. \& Scariot, A. Composição e estrutura da comunidade arbórea de uma Floresta Estacional Decidual sobre afloramento calcário no Brasil central. Revista Árvore, v. 28, n. 1, p. 69-75, 2004. DOI: 10.1590/S0100-67622004000100009.

Tropicos. Saint Louis: Missouri Botanical Garden, [2015]. Avaliable from: <http://www.tropicos.org/>. Acess on: 15 Mar. 2015. 
